Amanda Kay Lewton, MD, MSPH; Laura Elizabeth Morris, MD, MSPH

University of Missouri Department of Family \& Community Medicine, Columbia

DEPUTY EDITOR Katherine Hale, PharmD, BCPS, MFA

Department of Nursing, Heritage University, Toppenish, WA

doi: 10.12788/jfp.0309

\title{
Validated scoring system identifies low-risk syncope patients
}

\author{
This study validated the Canadian Syncope Risk Score \\ for predicting 30-day serious outcomes in patients \\ presenting to the ED within 24 hours of syncope.
}

\section{PRACTICE CHANGER}

Physicians should use the Canadian Syncope Risk Score (CSRS) to identify and send home very low- and low-risk patients from the emergency department (ED) after a syncopal episode.

\section{STRENGTH OF RECOMMENDATION}

A: Validated clinical decision rule based on a prospective cohort study ${ }^{1}$

Thiruganasambandamoorthy V, Sivilotti MLA, Le Sage N, et al. Multicenter emergency department validation of the Canadian Syncope Risk Score. JAMA Intern Med. 2020;180:737-744. doi:10.1001/ jamainternmed.2020.0288

\section{ILLUSTRATIVE CASE}

A 30-year-old woman presented to the ED after she "passed out" while standing at a concert. She lost consciousness for 10 seconds. After she revived, her friends drove her to the ED. She is healthy, with no chronic medical conditions, no medication use, and no drug or alcohol use. Should she be admitted to the hospital for observation?

0 yncope, a transient loss of consciousness followed by spontaneous complete recovery, accounts for $1 \%$ of ED visits. ${ }^{2}$ Approximately $10 \%$ of patients presenting to the ED will have a serious underlying condition identified and among $3 \%$ to $5 \%$ of these patients with syncope, the serious condition will be identified only after they leave the ED. ${ }^{1}$ Most patients have a benign course, but more than half of all patients presenting to the ED with syncope will be hospitalized, costing $\$ 2.4$ billion annually. ${ }^{2}$

Because of the high hospitalization rate of patients with syncope, a practical and accurate tool to risk-stratify patients is vital. Other tools, such as the San Francisco Syncope Rule, Short-Term Prognosis of Syncope, and Risk Stratification of Syncope in the Emergency Department, lack validation or are excessively complex, with extensive lab work or testing. ${ }^{3}$

The CSRS was previously derived from a large, multisite consecutive cohort, and was internally validated and reported according to the Transparent Reporting of a Multivariable Prediction Model for Individual Prognosis or Diagnosis guideline statement. ${ }^{4}$ Patients are assigned points based on clinical findings, test results, and the diagnosis given in the ED (TABLE $\left.{ }^{4}\right)$. The scoring system is used to stratify patients as very low $(-3,-2)$, low $(-1,0)$, medium $(1,2,3)$, high $(4,5)$, or very high $(\geq 6)$ risk. $^{4}$

\section{STUDY SUMMARY}

Less than $1 \%$ of very low- and low-risk patients had serious 30-day outcomes

This multisite Canadian prospective validation cohort study enrolled patients age $\geq 16$ years who presented to the ED within 24 hours of syncope. Both discharged and hospitalized patients were included. ${ }^{1}$

Patients were excluded if they had loss of consciousness for $>5$ minutes, mental status 
TABLE

Canadian Syncope Risk Score components ${ }^{4}$

\begin{tabular}{l|l}
\hline Category & Points \\
\hline Clinical evaluation & \\
\hline Predisposition to vasovagal symptoms & -1 \\
\hline History of heart disease & 1 \\
\hline Any systolic pressure reading $<90$ or $>180 \mathrm{~mm} \mathrm{Hg}$ & 2 \\
\hline Investigations & \\
\hline Elevated troponin level $\left(>99\right.$ th $^{-}$percentile of normal population) & 2 \\
\hline Abnormal QRS axis $\left(<-30^{\circ}\right.$ or $\left.>100^{\circ}\right)$ & 1 \\
\hline QRS duration $>130 \mathrm{~ms}$ & 1 \\
\hline Corrected QT interval $>480 \mathrm{~ms}$ & 2 \\
\hline Diagnosis in ED & \\
\hline Vasovagal syncope & -2 \\
\hline Cardiac syncope & 2 \\
\hline Total score (-3 to 11) & \\
\hline
\end{tabular}

ED, emergency department.

changes at presentation, history of current or previous seizure, or head trauma resulting in loss of consciousness. Patients requiring hospitalization secondary to trauma or those from whom an accurate history could not be obtained (eg, intoxication) were excluded, as were patients with a serious underlying condition identified during the original ED evaluation.

ED physicians confirmed patient eligibility, obtained verbal consent, and completed the data collection form. In addition, research assistants sought to identify eligible patients who were not previously enrolled by reviewing all ED visits during the study period.

To examine 30-day outcomes, researchers reviewed all available patient medical records, including administrative health records at all hospitals within the province; performed a telephone follow-up immediately after 30 days; and if no other information was found, searched the coroner's database. Two ED physicians (with a third resolving disagreements) determined if a serious outcome occurred, including any arrhythmia, intervention to treat arrythmia, death due to an unknown cause, myocardial infarction, structural heart disease, aortic dissection, pulmonary embolism, severe pulmonary hypertension, significant hemor- rhage, or subarachnoid hemorrhage. ${ }^{1}$

A total of 4131 patients made up the validation cohort. A serious condition was identified during the initial ED visit in 160 patients (3.9\%), who were excluded from the study, and 152 patients $(3.7 \%)$ were lost to followup. Of the 3819 patients included in the final analysis, troponin was not measured in 1566 patients (41\%), and an electrocardiogram was not obtained in 114 patients (3\%). A serious outcome within 30 days was experienced by 139 patients (3.6\%; 95\% CI, $3.1 \%-4.3 \%$ ). There was good correlation to the model-predicted serious outcome probability of $3.2 \%$ (95\% CI, $2.7 \%-3.8 \%$ ). ${ }^{1}$

Three of $1631(0.2 \%)$ patients classified as very low risk and 9 of $1254(0.7 \%)$ lowrisk patients experienced a serious outcome, and no patients died. In the group classified as medium risk, 55 of $687(8 \%)$ patients experienced a serious outcome, and there was 1 death. In the high-risk group, 32 of 167 (19.2\%) patients experienced a serious outcome, and there were 5 deaths. In the group classified as very high risk, 40 of $78(51.3 \%)$ patients experienced a serious outcome, and there were 7 deaths. The CSRS was able to identify very low- or low-risk patients (score of -1 or better) with a sensitivity of $97.8 \%$ (95\% CI, 93.8\%-99.6\%) and a specificity of $44.3 \%$ ( $95 \%$ CI, $42.7 \%-45.9 \%$ ). ${ }^{1}$

CONTINUED 


\section{WHAT"S NEW}

\section{This scoring system offers a validated method to risk-stratify ED patients}

Previous recommendations from the American College of Cardiology/American Heart Association suggested determining disposition of ED patients by using clinical judgment based on a list of risk factors such as age, chronic conditions, and medications. However, there was no scoring system. ${ }^{3}$ This new scoring system allows physicians to send home very low- and low-risk patients with reassurance that the likelihood of a serious outcome is less than $1 \%$. High-risk and very high-risk patients should be admitted to the hospital for further evaluation. Most moderate-risk patients ( $8 \%$ risk of serious outcome but $0.1 \%$ risk of death) can also be discharged after providers have a risk/benefit discussion, including precautions for signs of arrhythmia or need for urgent return to the hospital.

\section{CAVEATS}

\section{The study does not translate}

\section{to all clinical settings}

Because this study was done in EDs, the scoring system cannot necessarily be applied to urgent care or outpatient settings. However, $41 \%$ of the patients in the study did not have troponin testing performed. Therefore, physicians could consider using the scoring system in settings where this lab test is not immediately available.

This scoring system was also only validated with adult patients presenting within 24 hours of their syncopal episode. It is unknown how it may predict the outcomes of patients who present $>24$ hours after syncope.

\section{CHALLENGES TO IMPLEMENTATION}

\section{Clinicians may not be aware of the CSRS scoring system}

The main challenge to implementation is practitioner awareness of the CSRS scoring system and how to use it appropriately, as there are several different syncopal scoring systems that may already be in use. Additionally, depending on the electronic health record used, the CSRS scoring system may not be embedded. Using and documenting scores may also be a challenge.

\section{ACKNOWLEDGEMENT}

The PURLs Surveillance System was supported in part by Grant Number UL1RR024999 from the National Center for Research Resources, a Clinical Translational Science Award to the University of Chicago. The content is solely the responsibility of the authors and does not necessarily represent the official views of the National Center for Research Resources or the National Institutes of Health.

Copyright @ 2021 . The Family Physicians Inquiries Network. All rights reserved.

\section{References}

1. Thiruganasambandamoorthy V, Sivilotti MLA, Le Sage N, et al. Multicenter emergency department validation of the Canadian Syncope Risk Score. JAMA Intern Med. 2020;180:737-744. doi:10.1001/jamainternmed.2020.0288

2. Probst MA, Kanzaria HK, Gbedemah M, et al. National trends in resource utilization associated with $\mathrm{ED}$ visits for syncope. $\mathrm{Am}$ JEmerg Med. 2015;33:998-1001. doi:10.1016/j.ajem.2015.04.030

3. Shen WK, Sheldon RS, Benditt DG, et al. 2017 ACC/AHA/HRS guideline for the evaluation and management of patients with syncope: executive summary: a report of the American College of Cardiology/American Heart Association Task Force on Clinical Practice Guidelines and the Heart Rhythm Society. J Am Coll Cardiol. 2017;70:620-663. doi:10.1016/j.jacc.2017.03.002

4. Thiruganasambandamoorthy V, Kwong K, Wells GA, et al. Development of the Canadian Syncope Risk Score to predict serious adverse events after emergency department assessment of syncope. CMAJ. 2016;188:E289-E298. doi:10.1503/cmaj.151469

\section{The first mobile job board for Physicians, NPs, and PAs}

\section{Mobile Job Searches-access MedJobNetwork.com on the go from your smartphone or tablet}

Advanced Search Capabilities - search for jobs by specialty, job title, geographic location, employer, and more

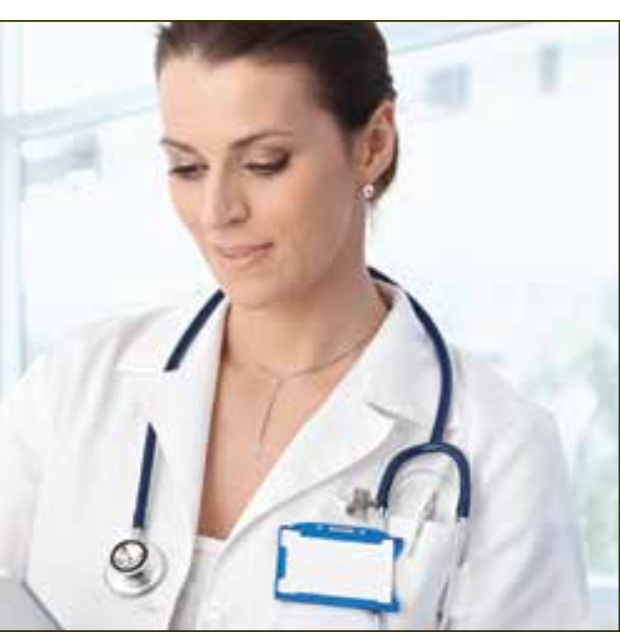

